\title{
Sharp inequalities and asymptotic expansion associated with the Wallis sequence
}

\author{
Ji-En Deng, Tao Ban* and Chao-Ping Chen
}

\section{"Correspondence:}

bantao454000@sohu.com

School of Mathematics and

Informatics, Henan Polytechnic

University, Jiaozuo, Henan 454000,

People's Republic of China

\begin{abstract}
We present asymptotic expansion of function involving the ratio of gamma functions and provide a recurrence relation for determining the coefficients of the asymptotic expansion. As a consequence, we obtain asymptotic expansion of the Wallis sequence. Also, we establish sharp inequalities for the Wallis sequence.
\end{abstract}

MSC: Primary 40A05; secondary 33B15; 41A60; 26D15

Keywords: Wallis sequence; gamma function; psi function; polygamma function; inequality; asymptotic expansion

\section{Introduction}

The Wallis sequence to which the title refers is

$$
W_{n}=\prod_{k=1}^{n} \frac{4 k^{2}}{4 k^{2}-1}, \quad n \in \mathbb{N}:=\{1,2,3, \ldots\}
$$

Wallis (1616-1703) discovered that

$$
\prod_{k=1}^{\infty} \frac{4 k^{2}}{4 k^{2}-1}=\frac{2}{1} \frac{2}{3} \frac{4}{3} \frac{4}{5} \frac{6}{5} \frac{6}{7} \frac{8}{7}=\cdots=\frac{\pi}{2}
$$

(see [1], p.68). Several elementary proofs of (1.2) can be found (see, for example, [2-4]). An interesting geometric construction produces (1.2) [5]. Many formulas exist for the representation of $\pi$, and a collection of these formulas is listed [6,7]. For more history of $\pi$ see $[1,8-10]$.

Some inequalities and asymptotic formulas associated with the Wallis sequence $W_{n}$ can be found (see, for example, [11-24]). Hirschhorn [13] proved that for $n \in \mathbb{N}$,

$$
\frac{\pi}{2}\left(1-\frac{1}{4 n+\frac{7}{3}}\right)<W_{n}<\frac{\pi}{2}\left(1-\frac{1}{4 n+\frac{8}{3}}\right) .
$$

Also in [13], Hirschhorn pointed out that if the $c_{j}$ are given by

$$
\tanh \left(\frac{x}{4}\right)=\sum_{j=0}^{\infty} c_{j} \frac{x^{2 j+1}}{(2 j) !}
$$

\section{Springer}


then, as $n \rightarrow \infty$,

$$
W_{n} \sim \frac{\pi}{2}\left(1+\frac{1}{2 n}\right)^{-1} \prod_{j \geq 0} \exp \left(\frac{c_{j}}{n^{2 j+1}}\right)=\frac{\pi}{2}\left(1+\frac{1}{2 n}\right)^{-1} \exp \left(\sum_{j=0}^{\infty} \frac{c_{j}}{n^{2 j+1}}\right)
$$

Very recently, Lin et al. [17] found that

$$
c_{j}=\frac{\left(2^{2 j+2}-1\right) B_{2 j+2}}{2^{2 j+1}(2 j+1)(j+1)}, \quad j \in \mathbb{N}_{0}:=\mathbb{N} \cup\{0\},
$$

where $B_{n}\left(n \in \mathbb{N}_{0}\right)$ are the Bernoulli numbers defined by the following generating function:

$$
\frac{z}{e^{z}-1}=\sum_{n=0}^{\infty} B_{n} \frac{z^{n}}{n !}, \quad|z|<2 \pi
$$

Also in [17], Lin et al. derived

$$
W_{n}=\frac{\pi}{2}\left(1-\frac{1}{4 n+\frac{5}{2}}\right)^{1-\frac{3}{64 n^{2}}+\frac{3}{64 n^{3}}-\frac{23}{1,024 n^{4}}+O\left(n^{-5}\right)}, \quad n \rightarrow \infty .
$$

The gamma function is defined for $x>0$ by

$$
\Gamma(x)=\int_{0}^{\infty} t^{x-1} e^{-t} \mathrm{~d} t
$$

The logarithmic derivative of $\Gamma(x)$, denoted by $\psi(x)=\Gamma^{\prime}(x) / \Gamma(x)$, is called psi (or digamma) function, and $\psi^{(k)}(x)(k \in \mathbb{N})$ are called polygamma functions. These functions play an important role in various branches of mathematics as well as in physics and engineering. For the various properties of these functions, please refer to [25], pp.255-260.

Define the function $W(x)$ by

$$
W(x)=\frac{\pi}{2}\left(1+\frac{1}{2 x}\right)^{-1} \frac{1}{x}\left[\frac{\Gamma(x+1)}{\Gamma\left(x+\frac{1}{2}\right)}\right]^{2} .
$$

It is easy to see that

$$
W_{n}=W(n)
$$

The first aim of present paper is to establish sharp inequalities for $W_{n}$. More precisely, we determine the best possible constants $\alpha, \beta$, and $\mu$ such that the double inequalities

$$
\frac{\pi}{2}\left(1-\frac{1}{4 n+\alpha}\right)<W_{n} \leq \frac{\pi}{2}\left(1-\frac{1}{4 n+\beta}\right)
$$

and

$$
\frac{\pi}{2}\left(1-\frac{1}{4 n+\frac{5}{2}}\right)^{\lambda}<W_{n} \leq \frac{\pi}{2}\left(1-\frac{1}{4 n+\frac{5}{2}}\right)^{\mu}
$$


hold for all $n \in \mathbb{N}$. The second aim of present paper is to develop the formula (1.7) to produce a complete asymptotic expansion. More precisely, we provide a recurrence relation for determining the coefficients $r_{j}\left(j \in \mathbb{N}_{0}\right)$ such that

$$
W(x) \sim \frac{\pi}{2}\left(1-\frac{1}{4 x+\frac{5}{2}}\right)^{\sum_{j=0}^{\infty} r_{j} x^{-j}}, \quad x \rightarrow \infty .
$$

\section{Lemmas}

The following lemmas are required in our present investigation.

Lemma 1 ([26], Corollary 1) Let $m, n \in \mathbb{N}$. Then for $x>0$,

$$
\begin{aligned}
& \sum_{j=1}^{2 m}\left(1-\frac{1}{2^{2 j}}\right) \frac{2 B_{2 j}}{(2 j) !} \frac{(2 j+n-2) !}{x^{2 j+n-1}} \\
&<(-1)^{n}\left(\psi^{(n-1)}(x+1)-\psi^{(n-1)}\left(x+\frac{1}{2}\right)\right)+\frac{(n-1) !}{2 x^{n}} \\
& \quad<\sum_{j=1}^{2 m-1}\left(1-\frac{1}{2^{2 j}}\right) \frac{2 B_{2 j}}{(2 j) !} \frac{(2 j+n-2) !}{x^{2 j+n-1}}
\end{aligned}
$$

where $B_{n}$ are the Bernoulli numbers.

It follows from (2.1) that, for $x>0$,

$$
\frac{1}{2 x}-\frac{1}{8 x^{2}}+\frac{1}{64 x^{4}}-\frac{1}{128 x^{6}}<\psi(x+1)-\psi\left(x+\frac{1}{2}\right)<\frac{1}{2 x}-\frac{1}{8 x^{2}}+\frac{1}{64 x^{4}}
$$

and

$$
-\frac{1}{2 x^{2}}+\frac{1}{4 x^{3}}-\frac{1}{16 x^{5}}<\psi^{\prime}(x+1)-\psi^{\prime}\left(x+\frac{1}{2}\right)<-\frac{1}{2 x^{2}}+\frac{1}{4 x^{3}}-\frac{1}{16 x^{5}}+\frac{3}{64 x^{7}} .
$$

Lemma 2 For all $x \geq 1$,

$$
\left[\frac{\Gamma(x+1)}{\Gamma\left(x+\frac{3}{2}\right)}\right]^{2}<\frac{1}{x}-\frac{3}{4 x^{2}}+\frac{17}{32 x^{3}}-\frac{45}{128 x^{4}}+\frac{443}{2,048 x^{5}} .
$$

Proof We consider the function $G(x)$ defined by

$$
\begin{aligned}
G(x)= & 2 \ln \Gamma(x+1)-2\left[\ln \Gamma\left(x+\frac{1}{2}\right)+\ln \left(x+\frac{1}{2}\right)\right] \\
& -\ln \left(\frac{1}{x}-\frac{3}{4 x^{2}}+\frac{17}{32 x^{3}}-\frac{45}{128 x^{4}}+\frac{443}{2,048 x^{5}}\right) .
\end{aligned}
$$

From the asymptotic expansion ([25], p.257):

$$
\begin{aligned}
x^{b-a} \frac{\Gamma(x+a)}{\Gamma(x+b)}= & +\frac{(a-b)(a+b-1)}{2 x} \\
& +\frac{1}{12}\left(\begin{array}{c}
a-b \\
2
\end{array}\right)\left(3(a+b-1)^{2}-a+b-1\right) \frac{1}{x^{2}}+\cdots \quad \text { as } x \rightarrow \infty,
\end{aligned}
$$


we conclude that

$$
\lim _{x \rightarrow \infty} G(x)=0
$$

Differentiating and applying the first inequality in (2.2) yield, for $x \geq 1$,

$$
\begin{aligned}
G^{\prime}(x)= & 2\left[\psi(x+1)-\psi\left(x+\frac{1}{2}\right)\right] \\
& -\frac{4,096 x^{5}-2,048 x^{4}+896 x^{3}-384 x^{2}+222 x-2,215}{x(2 x+1)\left(2,048 x^{4}-1,536 x^{3}+1,088 x^{2}-720 x+443\right)} \\
> & 2\left(\frac{1}{2 x}-\frac{1}{8 x^{2}}+\frac{1}{64 x^{4}}-\frac{1}{128 x^{6}}\right) \\
& -\frac{4,096 x^{5}-2,048 x^{4}+896 x^{3}-384 x^{2}+222 x-2,215}{x(2 x+1)\left(2,048 x^{4}-1,536 x^{3}+1,088 x^{2}-720 x+443\right)} \\
= & \left(158,193+797,514(x-1)+1,606,106(x-1)^{2}+1,619,020(x-1)^{3}\right. \\
& \left.+816,432(x-1)^{4}+164,640(x-1)^{5}\right) /\left(64 x^{6}(2 x+1)(1,323+5,040(x-1)\right. \\
& \left.\left.+8,768(x-1)^{2}+6,656(x-1)^{3}+2,048(x-1)^{4}\right)\right) \\
> & 0
\end{aligned}
$$

This leads to

$$
\begin{aligned}
G(x) & =\ln \left[\frac{\Gamma(x+1)}{\Gamma\left(x+\frac{3}{2}\right)}\right]^{2}-\ln \left(\frac{1}{x}-\frac{3}{4 x^{2}}+\frac{17}{32 x^{3}}-\frac{45}{128 x^{4}}+\frac{443}{2,048 x^{5}}\right) \\
& <\lim _{x \rightarrow \infty} G(x)=0, \quad x \geq 1 .
\end{aligned}
$$

The proof of Lemma 2 is complete.

By (2.2), we obtain

$$
\begin{aligned}
(2 x+1)\left[\psi(x+1)-\psi\left(x+\frac{1}{2}\right)\right]-1 & <(2 x+1)\left(\frac{1}{2 x}-\frac{1}{8 x^{2}}+\frac{1}{64 x^{4}}\right)-1 \\
& =\frac{1}{4 x}-\frac{1}{8 x^{2}}+\frac{1}{32 x^{3}}+\frac{1}{64 x^{4}} .
\end{aligned}
$$

By (2.4), we get

$$
\begin{aligned}
1-\left(x+\frac{1}{2}\right)\left[\frac{\Gamma(x+1)}{\Gamma\left(x+\frac{3}{2}\right)}\right]^{2} & >1-\left(x+\frac{1}{2}\right)\left(\frac{1}{x}-\frac{3}{4 x^{2}}+\frac{17}{32 x^{3}}-\frac{45}{128 x^{4}}+\frac{443}{2,048 x^{5}}\right) \\
& =\frac{1}{4 x}-\frac{5}{32 x^{2}}+\frac{11}{128 x^{3}}-\frac{83}{2,048 x^{4}}-\frac{443}{4,096 x^{5}} .
\end{aligned}
$$

The proof of Theorem 1 makes use of (2.6) and (2.7).

Lemma 3 ([27]) Let $-\infty \leq a<b \leq \infty$. Let $f$ and $g$ be differentiable functions on an interval $(a, b)$. Assume that either $g^{\prime}>0$ everywhere on $(a, b)$ or $g^{\prime}<0$ on $(a, b)$. Suppose that $f(a+)=$ $g(a+)=0$ or $f(b-)=g(b-)=0$. Then 
(1) if $\frac{f^{\prime}}{g^{\prime}}$ is increasing on $(a, b)$, then $\left(\frac{f}{g}\right)^{\prime}>0$ on $(a, b)$;

(2) if $\frac{f^{\prime}}{g^{\prime}}$ is decreasing on $(a, b)$, then $\left(\frac{f}{g}\right)^{\prime}<0$ on $(a, b)$.

\section{Sharp inequalities}

Theorem 1 For all $n \in \mathbb{N}$,

$$
\frac{\pi}{2}\left(1-\frac{1}{4 n+\alpha}\right)<W_{n} \leq \frac{\pi}{2}\left(1-\frac{1}{4 n+\beta}\right)
$$

with the best possible constants

$$
\alpha=\frac{5}{2} \quad \text { and } \quad \beta=\frac{32-9 \pi}{3 \pi-8}=2.614909986 \ldots
$$

Equality in (3.1) occurs for $n=1$.

Proof The inequality (3.1) can be written as

$$
\alpha \leq F(n)<\beta
$$

where

$$
F(x)=\frac{1}{1-\frac{1}{x+1 / 2}\left[\frac{\Gamma(x+1)}{\Gamma(x+1 / 2)}\right]^{2}}-4 x .
$$

Using (2.5), we conclude that

$$
\lim _{x \rightarrow \infty} F(x)=\frac{5}{2}
$$

Differentiating $F(x)$ and applying (2.4), (2.6), and (2.7) yield, for $x \geq 6$,

$$
\begin{aligned}
(1- & \left.\left(x+\frac{1}{2}\right)\left[\frac{\Gamma(x+1)}{\Gamma\left(x+\frac{3}{2}\right)}\right]^{2}\right)^{2} F^{\prime}(x) \\
= & \left\{(2 x+1)\left[\psi(x+1)-\psi\left(x+\frac{1}{2}\right)\right]-1\right\}\left[\frac{\Gamma(x+1)}{\Gamma\left(x+\frac{3}{2}\right)}\right]^{2} \\
& -4\left(1-\left(x+\frac{1}{2}\right)\left[\frac{\Gamma(x+1)}{\Gamma\left(x+\frac{3}{2}\right)}\right]^{2}\right)^{2} \\
< & \left(\frac{1}{4 x}-\frac{1}{8 x^{2}}+\frac{1}{32 x^{3}}+\frac{1}{64 x^{4}}\right)\left(\frac{1}{x}-\frac{3}{4 x^{2}}+\frac{17}{32 x^{3}}-\frac{45}{128 x^{4}}+\frac{443}{2,048 x^{5}}\right) \\
& -4\left(\frac{1}{4 x}-\frac{5}{32 x^{2}}+\frac{11}{128 x^{3}}-\frac{83}{2,048 x^{4}}-\frac{443}{4,096 x^{5}}\right)^{2} \\
= & \frac{1}{4,194,304 x^{10}}\left(248,771,713+769,183,956(x-6)+510,154,660(x-6)^{2}\right. \\
& +149,038,464(x-6)^{3}+22,221,824(x-6)^{4} \\
& \left.+1,658,880(x-6)^{5}+49,152(x-6)^{6}\right) \\
< & 0 .
\end{aligned}
$$


Straightforward calculation produces

$$
\begin{aligned}
& F(1)=\frac{32-9 \pi}{3 \pi-8}=2.6149 \ldots, \\
& F(2)=\frac{-315 \pi+1,024}{45 \pi-128}=2.5724 \ldots, \\
& F(3)=\frac{-1,925 \pi+6,144}{175 \pi-512}=2.5526 \ldots, \\
& F(4)=\frac{-165,375 \pi+524,288}{11,025 \pi-32,768}=2.5412 \ldots, \\
& F(5)=\frac{-829,521 \pi+2,621,440}{43,659 \pi-131,072}=2.5338 \ldots, \\
& F(6)=\frac{-15,954,939 \pi+50,331,648}{693,693 \pi-2,097,152}=2.5286 \ldots
\end{aligned}
$$

Thus, the sequence $(F(n))_{n \in \mathbb{N}}$ is strictly decreasing. This leads to

$$
\frac{5}{2}<\lim _{x \rightarrow \infty} F(x)<F(n) \leq F(1)=\frac{32-9 \pi}{3 \pi-8}, \quad n \in \mathbb{N} .
$$

The proof of Theorem 1 is complete.

Remark 1 In fact, Elezović et al. [12] have previously shown that $\frac{5}{2}$ is the best possible constant for a lower bound of $W_{n}$ of the type $\frac{\pi}{2}\left(1-\frac{1}{4 n+\alpha}\right)$. Moreover, the authors pointed out that

$$
W_{n}=\frac{\pi}{2}\left(1-\frac{1}{4 n+\frac{5}{2}}\right)+O\left(\frac{1}{n^{3}}\right), \quad n \rightarrow \infty
$$

Theorem 2 For all $n \in \mathbb{N}$,

$$
\frac{\pi}{2}\left(1-\frac{1}{4 n+\frac{5}{2}}\right)^{\lambda}<W_{n} \leq \frac{\pi}{2}\left(1-\frac{1}{4 n+\frac{5}{2}}\right)^{\mu}
$$

with the best possible constants

$$
\lambda=1 \quad \text { and } \quad \mu=\frac{\ln (3 \pi / 8)}{\ln (13 / 11)}=0.98112316 \ldots
$$

Equality in (3.2) occurs for $n=1$.

Proof Inequality (3.2) can be written as

$$
\lambda>x_{n} \geq \mu,
$$

where the sequence $\left(x_{n}\right)_{n \in \mathbb{N}}$ is defined by

$$
x_{n}=\frac{\ln \left(\frac{1}{n+\frac{1}{2}}\left(\frac{\Gamma(n+1)}{\Gamma\left(n+\frac{1}{2}\right)}\right)^{2}\right)}{\ln \left(1-\frac{1}{4 n+\frac{5}{2}}\right)} .
$$


We are now in a position to show that the sequence $\left(x_{n}\right)_{n \in \mathbb{N}}$ is strictly increasing. To this end, we consider the function $f(x)$ defined by

$$
f(x)=\frac{2 \ln \Gamma(x+1)-2 \ln \Gamma\left(x+\frac{1}{2}\right)-\ln \left(x+\frac{1}{2}\right)}{\ln \left(1-\frac{1}{4 x+\frac{5}{2}}\right)}=\frac{f_{1}(x)}{f_{2}(x)},
$$

where

$$
f_{1}(x)=2 \ln \Gamma(x+1)-2 \ln \Gamma\left(x+\frac{1}{2}\right)-\ln \left(x+\frac{1}{2}\right)
$$

and

$$
f_{2}(x)=\ln \left(1-\frac{1}{4 x+\frac{5}{2}}\right)
$$

We conclude from the asymptotic formula of $\ln \Gamma(z)$ ([25], p.257) that

$$
f_{1}(\infty)=\lim _{x \rightarrow \infty} f_{1}(x)=0
$$

Elementary calculations show that

$$
\frac{8 f_{1}^{\prime}(x)}{f_{2}^{\prime}(x)}=\left(64 x^{2}+64 x+15\right)\left[\psi(x+1)-\psi\left(x+\frac{1}{2}\right)-\frac{1}{2 x+1}\right]=: f_{3}(x) .
$$

By using inequalities (2.2) and (2.3), we obtain, for $x \geq 2$,

$$
\begin{aligned}
f_{3}^{\prime}(x)= & (128 x+64)\left[\psi(x+1)-\psi\left(x+\frac{1}{2}\right)-\frac{1}{2 x+1}\right] \\
& +\left(64 x^{2}+64 x+15\right)\left[\psi^{\prime}(x+1)-\psi^{\prime}\left(x+\frac{1}{2}\right)+\frac{2}{(2 x+1)^{2}}\right] \\
> & (128 x+64)\left[\frac{1}{2 x}-\frac{1}{8 x^{2}}+\frac{1}{64 x^{4}}-\frac{1}{128 x^{6}}-\frac{1}{2 x+1}\right] \\
& +\left(64 x^{2}+64 x+15\right)\left[-\frac{1}{2 x^{2}}+\frac{1}{4 x^{3}}-\frac{1}{16 x^{5}}+\frac{2}{(2 x+1)^{2}}\right] \\
= & \frac{202+4,881(x-2)+7,860(x-2)^{2}+4,896(x-2)^{3}+1,368(x-2)^{4}+144(x-2)^{5}}{16 x^{6}(2 x+1)^{2}} \\
> & 0 .
\end{aligned}
$$

Hence, $f_{3}(x)$ and $\frac{f_{1}^{\prime}(x)}{f_{2}^{\prime}(x)}$ are both strictly increasing for $x \geq 2$. By Lemma 3 , the function

$$
f(x)=\frac{f_{1}(x)}{f_{2}(x)}=\frac{f_{1}(x)-f_{1}(\infty)}{f_{2}(x)-f_{2}(\infty)}
$$

is strictly increasing for $x \geq 2$. Therefore, the sequence $\left(x_{n}\right)$ is strictly increasing for $n \geq 2$. Direct computation would yield

$$
x_{1}=\frac{\ln (3 \pi / 8)}{\ln (13 / 11)}=0.9811 \ldots, \quad x_{2}=\frac{-7 \ln 2+2 \ln 3+\ln \pi+\ln 5}{-\ln 19+\ln 3+\ln 7}=0.9927 \ldots
$$


Consequently, the sequence $\left(x_{n}\right)_{n \in \mathbb{N}}$ is strictly increasing. This leads to

$$
\lim _{n \rightarrow \infty} x_{n}>x_{n} \geq x_{1}=\frac{\ln (3 \pi / 8)}{\ln (13 / 11)} \quad \text { for } n \in \mathbb{N} \text {. }
$$

It remains to prove that

$$
\lim _{n \rightarrow \infty} x_{n}=1
$$

We conclude from the asymptotic formula of $\ln \Gamma(z)([25], \mathrm{p} .257)$ that

$$
f(x)=\frac{1+O\left(x^{-1}\right)}{1+O\left(x^{-1}\right)} \rightarrow 1 \quad \text { as } x \rightarrow \infty
$$

which implies (3.3). This completes the proof of Theorem 2.

\section{Asymptotic expansion}

Theorem 3 The function $W(x)$, defined by (1.8), has the following asymptotic expansion:

$$
W(x) \sim \frac{\pi}{2}\left(1-\frac{1}{4 x+\frac{5}{2}}\right)^{\sum_{j=0}^{\infty} r_{j} x^{-j}}, \quad x \rightarrow \infty,
$$

with the coefficients $r_{j}$ given by the recurrence relation

$$
r_{0}=1, \quad r_{j}=4 \sum_{k=0}^{j-1} r_{k} q_{j-k+1}-4 p_{j+1}, \quad j \in \mathbb{N},
$$

where

$$
p_{j}=(-1)^{j-1}\left(-\frac{1}{j 2^{j}}+\frac{2\left((-1)^{j+1}-\left(2^{-j}-1\right)\right) B_{j+1}}{j(j+1)}\right), \quad j \in \mathbb{N}
$$

and

$$
q_{j}=-\sum_{k=0}^{j-1} \frac{1}{(k+1) \cdot 4^{k+1}}\left(\begin{array}{c}
j-1 \\
j-k-1
\end{array}\right)\left(-\frac{5}{8}\right)^{j-k-1}, \quad j \in \mathbb{N} .
$$

Here, $B_{n}$ are the Bernoulli numbers.

Proof Write (4.1) as

$$
\frac{\ln \left(\frac{2}{\pi} W(x)\right)}{\ln \left(1-\frac{1}{4 x+\frac{5}{2}}\right)} \sim \sum_{j=0}^{\infty} \frac{r_{j}}{x^{j}}, \quad x \rightarrow \infty .
$$

The logarithm of gamma function has asymptotic expansion (see [28], p.32):

$$
\ln \Gamma(x+t) \sim\left(x+t-\frac{1}{2}\right) \ln x-x+\frac{1}{2} \ln (2 \pi)+\sum_{n=1}^{\infty} \frac{(-1)^{n+1} B_{n+1}(t)}{n(n+1)} \frac{1}{x^{n}}
$$


as $x \rightarrow \infty$, where $B_{n}(t)$ denotes the Bernoulli polynomials defined by the following generating function:

$$
\frac{x e^{t x}}{e^{x}-1}=\sum_{n=0}^{\infty} B_{n}(t) \frac{x^{n}}{n !}
$$

From (4.6), we obtain, as $x \rightarrow \infty$,

$$
\left[\frac{\Gamma(x+t)}{\Gamma(x+s)}\right]^{1 /(t-s)} \sim x \exp \left(\frac{1}{t-s} \sum_{j=1}^{\infty} \frac{(-1)^{j+1}\left(B_{j+1}(t)-B_{j+1}(s)\right)}{j(j+1)} \frac{1}{x^{j}}\right) .
$$

Setting $(s, t)=\left(\frac{1}{2}, 1\right)$ in $(4.7)$ and noting that

$$
B_{n}(0)=(-1)^{n} B_{n}(1)=B_{n} \quad \text { and } \quad B_{n}\left(\frac{1}{2}\right)=\left(2^{1-n}-1\right) B_{n} \quad \text { for } n \in \mathbb{N}_{0}
$$

(see [25], p.805), we obtain, as $x \rightarrow \infty$,

$$
\left[\frac{\Gamma(x+1)}{\Gamma\left(x+\frac{1}{2}\right)}\right]^{2} \sim x \exp \left(\sum_{j=1}^{\infty} \frac{2\left(1-(-1)^{j+1}\left(2^{-j}-1\right)\right) B_{j+1}}{j(j+1)} \frac{1}{x^{j}}\right) .
$$

By using the Maclaurin expansion of $\ln (1+t)$,

$$
\ln (1+t)=\sum_{j=1}^{\infty} \frac{(-1)^{j-1}}{j} t^{j} \quad \text { for }-1<t \leq 1,
$$

we obtain

$$
\left(1+\frac{1}{2 x}\right)^{-1} \sim \exp \left(\sum_{j=1}^{\infty} \frac{(-1)^{j}}{j^{j}} \frac{1}{x^{j}}\right) \quad \text { as } x \rightarrow \infty
$$

Applying (4.8) and (4.9) yields

$$
\ln \left(\frac{2}{\pi} W(x)\right) \sim \sum_{j=1}^{\infty} \frac{p_{j}}{x^{j}}, \quad x \rightarrow \infty
$$

with

$$
p_{j}=(-1)^{j-1}\left(-\frac{1}{j 2^{j}}+\frac{2\left((-1)^{j+1}-\left(2^{-j}-1\right)\right) B_{j+1}}{j(j+1)}\right), \quad j \in \mathbb{N} .
$$

The Maclaurin expansion of $\ln (1+t)$ with $t=-\frac{1}{4 x+\frac{5}{2}}$, yields

$$
\begin{aligned}
\ln \left(1-\frac{1}{4 x+\frac{5}{2}}\right) & \sim-\sum_{j=1}^{\infty} \frac{1}{j \cdot 4^{j} x^{j}}\left(1+\frac{5}{8 x}\right)^{-j} \\
& \sim-\sum_{j=1}^{\infty} \frac{1}{j \cdot 4^{j} x^{j}} \sum_{k=0}^{\infty}\left(\begin{array}{c}
-j \\
k
\end{array}\right) \frac{5^{k}}{8^{k} x^{k}}
\end{aligned}
$$




$$
\begin{aligned}
& \sim-\sum_{j=1}^{\infty} \frac{1}{j \cdot 4^{j} x^{j}} \sum_{k=0}^{\infty}(-1)^{k}\left(\begin{array}{c}
k+j-1 \\
k
\end{array}\right) \frac{5^{k}}{8^{k} x^{k}} \\
& \sim-\sum_{j=1}^{\infty} \sum_{k=0}^{j-1} \frac{1}{(k+1) \cdot 4^{k+1}}\left(\begin{array}{c}
j-1 \\
j-k-1
\end{array}\right)\left(-\frac{5}{8}\right)^{j-k-1} \frac{1}{x^{j}} .
\end{aligned}
$$

That is,

$$
\ln \left(1-\frac{1}{4 x+\frac{5}{2}}\right) \sim \sum_{j=1}^{\infty} \frac{q_{j}}{x^{j}}
$$

with

$$
q_{j}=-\sum_{k=0}^{j-1} \frac{1}{(k+1) \cdot 4^{k+1}}\left(\begin{array}{c}
j-1 \\
j-k-1
\end{array}\right)\left(-\frac{5}{8}\right)^{j-k-1}, \quad j \in \mathbb{N} .
$$

It follows from (4.5) that

$$
\begin{aligned}
& \frac{\sum_{j=1}^{\infty} p_{j} x^{-j}}{\sum_{j=1}^{\infty} q_{j} x^{-j}} \sim \sum_{j=0}^{\infty} \frac{r_{j}}{x^{j}}, \\
& \sum_{j=1}^{\infty} \frac{p_{j}}{x^{j}} \sim \sum_{j=0}^{\infty} \frac{r_{j}}{x^{j}} \sum_{k=1}^{\infty} \frac{q_{k}}{x^{k}}, \\
& \sum_{j=1}^{\infty} \frac{p_{j}}{x^{j}} \sim \sum_{j=1}^{\infty}\left(\sum_{k=0}^{j-1} r_{k} q_{j-k}\right) \frac{1}{x^{j}} .
\end{aligned}
$$

We then obtain

$$
\begin{aligned}
& p_{j}=\sum_{k=0}^{j-1} r_{k} q_{j-k}, \quad j \in \mathbb{N}, \\
& p_{j}=\sum_{k=0}^{j-2} r_{k} q_{j-k}+r_{j-1} q_{1}, \quad j \geq 2 .
\end{aligned}
$$

Noting that $q_{1}=-\frac{1}{4}$, we obtain

$$
r_{j-1}=4 \sum_{k=0}^{j-2} r_{k} q_{j-k}-4 p_{j}, \quad j \geq 2,
$$

and an empty sum (as usual) is understood to be nil. Noting that $p_{1}=-\frac{1}{4}$, we then obtain the recurrence relation

$$
r_{0}=1, \quad r_{j}=4 \sum_{k=0}^{j-1} r_{k} q_{j-k+1}-4 p_{j+1}, \quad j \in \mathbb{N} .
$$

The proof of Theorem 3 is complete. 
Here, from (4.1), we give the following explicit asymptotic expansion:

$$
W_{n}=\frac{\pi}{2}\left(1-\frac{1}{4 n+\frac{5}{2}}\right)^{1-\frac{3}{64 n^{2}}+\frac{3}{64 n^{3}}-\frac{23}{1,024 n^{4}}-\frac{1}{512 n^{5}}+\cdots}, \quad n \rightarrow \infty
$$

which develops the formula (1.7) to produce a complete asymptotic expansion.

\section{Competing interests}

The authors declare that they have no competing interests.

\section{Authors' contributions}

All authors read and approved the final manuscript.

\section{Acknowledgements}

The authors thank the referees for their careful reading of the manuscript and insightful comments.

\section{Received: 2 January 2015 Accepted: 18 May 2015 Published online: 10 June 2015}

\section{References}

1. Berggren, L, Borwein, J, Borwein, P (eds.): Pi: A Source Book, 2nd edn. Springer, New York (2000)

2. Amdeberhan, T, Espinosa, O, Moll, VH, Straub, A: Wallis-Ramanujan-Schur-Feynman. Am. Math. Mon. 117, 618-632 (2010)

3. Miller, SJ: A probabilistic proof of Wallis' formula for $\pi$. Am. Math. Mon. 115, 740-745 (2008)

4. Wästlund, J: An elementary proof of the Wallis product formula for pi. Am. Math. Mon. 114, 914-917 (2007)

5. Myerson, G: The limiting shape of a sequence of rectangles. Am. Math. Mon. 99, 279-280 (1992)

6. Sofo, A: Some representations of $\pi$. Aust. Math. Soc. Gaz. 31, 184-189 (2004)

7. Sofo, A: $\pi$ and some other constants. J. Inequal. Pure Appl. Math. 6(5), Article 138 (2005) (electronic)

8. Beckmann, P: A History of Pi. St. Martin's, New York (1971)

9. Dunham, W: Journey Through Genius: The Great Theorems of Mathematics. Penguin, Baltimore (1990)

10. Agarwal, RP, Agarwal, H, Sen, SK: Birth, growth and computation of pi to ten trillion digits. Adv. Differ. Equ. 2013, 100 (2013). doi:10.1186/1687-1847-2013-100

11. Burić, T, Elezović, N, Šimić, R: Asymptotic expansions of the multiple quotients of gamma functions with applications. Math. Inequal. Appl. 16, 1159-1170 (2013)

12. Elezović, N, Lin, L, Vukšić, L: Inequalities and asymptotic expansions for the Wallis sequence and the sum of the Wallis ratio. J. Math. Inequal. 7, 679-695 (2013)

13. Hirschhorn, MD: Comments on the paper: 'Wallis sequence estimated through the Euler-Maclaurin formula: even from the Wallis product $\pi$ could be computed fairly accurately' by V. Lampret. Aust. Math. Soc. Gaz. 32, 194 (2005)

14. Lampret, $\mathrm{V}$ : Wallis sequence estimated through the Euler-Maclaurin formula: even from the Wallis product $\pi$ could be computed fairly accurately. Aust. Math. Soc. Gaz. 31, 328-339 (2004)

15. Lampret, V: An asymptotic approximation of Wallis' sequence. Cent. Eur. J. Math. 10, 775-787 (2012)

16. Lin, L: Further refinements of Gurland's formula for $\pi$. J. Inequal. Appl. 2013, 48 (2013)

17. Lin, L, Deng, J-E, Chen, C-P: Inequalities and asymptotic expansions associated with the Wallis sequence. J. Inequal. Appl. 2014, 251 (2014)

18. Mortici, C: Estimating $\pi$ from the Wallis sequence. Math. Commun. 17, 489-495 (2012)

19. Mortici, C, Cristea, VG, Lu, D: Completely monotonic functions and inequalities associated to some ratio of gamma function. Appl. Math. Comput. 240, 168-174 (2014)

20. Mortici, C: Completely monotone functions and the Wallis ratio. Appl. Math. Lett. 25, 717-722 (2012)

21. Mortici, C: Sharp inequalities and complete monotonicity for the Wallis ratio. Bull. Belg. Math. Soc. Simon Stevin 17, 929-936 (2010)

22. Mortici, C: A new method for establishing and proving new bounds for the Wallis ratio. Math. Inequal. Appl. 13, 803-815 (2010)

23. Mortici, C: New approximation formulas for evaluating the ratio of gamma functions. Math. Comput. Model. $\mathbf{5 2}$ 425-433 (2010)

24. Păltănea, E: On the rate of convergence of Wallis' sequence. Aust. Math. Soc. Gaz. 34, 34-38 (2007)

25. Abramowitz, M, Stegun, IA (eds.): Handbook of Mathematical Functions with Formulas, Graphs, and Mathematical Tables, 9th edn. National Bureau of Standards: Applied Mathematics Series, vol. 55. Dover, New York (1972)

26. Chen, CP, Paris, RB: Inequalities, asymptotic expansions and completely monotonic functions related to the gamma function. Appl. Math. Comput. 250, 514-529 (2015)

27. Pinelis, I: L'Hospital type rules for monotonicity, with applications. J. Inequal. Pure Appl. Math. 3(1), Article 5 (2002) (electronic)

28. Luke, YL: The Special Functions and Their Approximations, vol. I. Academic Press, New York (1969) 